\title{
Immature stages of Galagete protozona (Lepidoptera: Gelechioidea: Autostichidae) from the Galapagos Islands: description and notes on biology
}

\author{
Patrick Schmitz ${ }^{1}$ \\ Muséum d'histoire naturelle, C.P. 6434, CH-1211 Geneva 6, Switzerland; Département de \\ Zoologie et Biologie Animale, Molecular Systematics Group, Université de Genève, \\ 30 quai Ernest Ansermet, CH-1211 Geneva 4, Switzerland \\ Bernard Landry \\ Muséum d'histoire naturelle, C.P. 6434, CH-1211 Geneva 6, Switzerland
}

\begin{abstract}
The morphology of the larva and pupa of Galagete protozona (Meyrick), an endemic of the Galapagos Islands, is described and illustrated. The immatures were observed feeding within droppings of the land iguana Conolophus subcristatus (Gray) (Iguanidae) on the island of Fernandina in 2005.
\end{abstract}

Résumé-La morphologie de la larve ainsi que de la chrysalide de Galagete protozona (Meyrick), endémique des îles Galapagos, est décrite et illustrée. Les immatures ont été observés se nourrissant dans les excréments de l'iguane terrestre Conolophus subcristatus (Gray) (Iguanidae) sur l'île de Fernandina en 2005.

\section{Introduction}

Galagete protozona (Meyrick, 1926) was described in the genus Gelechia Hübner (Lepidoptera: Gelechiidae) on the basis of three specimens from the Galapagos Islands. Landry (2002) transferred the species to the genus Galagete in the Autostichidae and provided a redescription of the adult. This genus, with a total of 12 species, represents an endemic insular radiation comparable in size to that of Darwin's finches. This group of microlepidoptera is a distinctive element of the moth fauna of the Galapagos Islands because it represents the largest endemic radiation of Lepidoptera in the archipelago and is present on all major islands at almost all elevations (Landry 2002). Several specimens of Galagete darwini Landry, 2002 and Galagete levequei Landry, 2002 were reared from dead leaves and branches of Scalesia species, an endemic genus of the Asteraceae, and one specimen of Galagete gnathodoxa (Meyrick, 1926) was reared from the droppings of the endemic
Galapagos tortoise, Geochelone nigra (Quoy and Gaimard, 1824) (Testudinidae) (Landry 2002). However, the immature stages have not been preserved or studied. Here, the morphology of the larva and pupa and the scavenger feeding habit of the larva of $G$. protozona are described.

\section{Material and methods}

This study is based on 67 larvae and 4 pupae of G. protozona preserved in $70 \%$ ethanol after being collected at elevations of 353 to $815 \mathrm{~m}$ in February 2005 on the island of Fernandina, the youngest and most active volcano in the Galapagos Archipelago. The arid slopes of the island are home to an undisturbed and large population of the endemic Galapagos land iguana Conolophus subcristatus (Gray, 1831) (Iguanidae). Droppings of these iguanas were found with numerous small holes in them. It was later discovered that these holes were the result of the activity of larvae of G. protozona.

Received 27 September 2006. Accepted 30 November 2006.

${ }^{1}$ Corresponding author (e-mail: patrick.schmitz@ville-ge.ch). 
Fig. 1. Adults of Galagete protozona just after emergence from droppings of Galapagos land iguanas (Conolophus subcristatus) collected on the island of Fernandina.

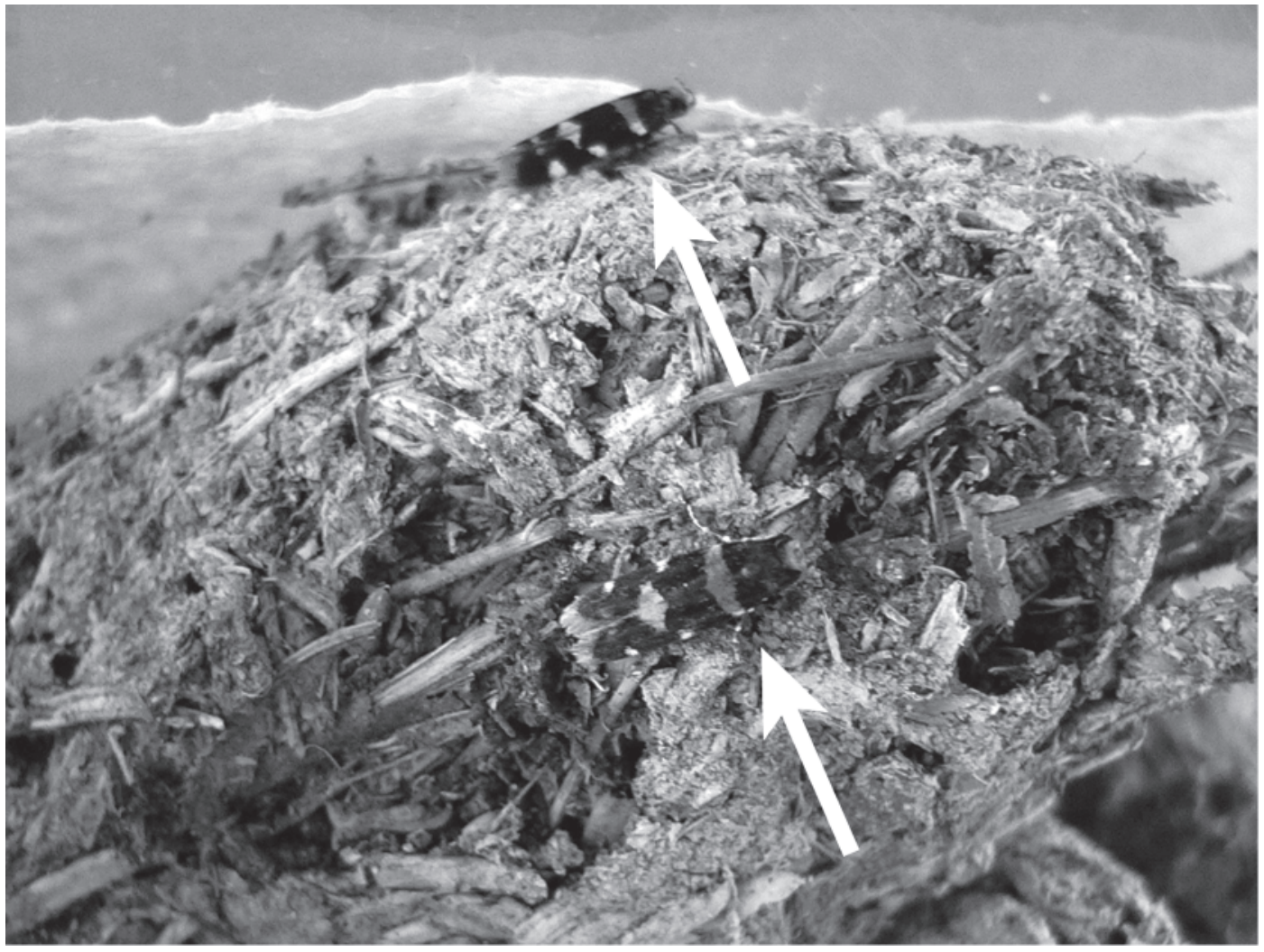

Many adults of G. protozona were reared from these droppings (Fig. 1), and immature stages were extracted, preserved, and studied.

For the electron microscopy study, larvae were cleaned in $10 \%$ ethanol with a hairbrush and dehydrated in increasing concentrations of ethanol to absolute ethanol. After dehydration, specimens were critical-point dried using a SPIDRYTM critical-point dryer (Structure Probe Inc., West Chester, Pennsylvania), mounted on stubs, and coated with gold-palladium (40\%$60 \%$ ) using a Cressington sputter coater (Cressington Scientific Instruments Ltd., Watford, England). Images were taken with a Zeiss DSM940A scanning electron microscope.

Gross morphological observations and measurements of the larvae and pupae were made using a dissecting microscope (reflected light) with a calibrated micrometer. Maps of the larval chaetotaxy and pupae were initially drawn using a camera lucida mounted on a Wild compound microscope. Terminology for chaetotaxy follows Stehr (1987). All vouchers of
G. protozona (larvae, pupae, and reared adult specimens) are deposited in the Natural History Museum of Geneva (MHNG), Geneva, Switzerland.

To certify that the larvae corresponded to the adults found, we sequenced a fragment of the mitochondrial cytochrome oxidase I (COI) gene. Whole genomic DNA was extracted using the NucleoSpin ${ }^{\circledR}$ kit (Macherey-Nagel, Düren, Germany). The COI gene was amplified by PCR with two primers: Jerry (5'-CAACATTTATTTTGATTTTTTGG-3') and Pat2 (5'-TCCATTACATATAATCTGCCATATTAG-3'). The thermal profile consisted of an initial denaturation at $95{ }^{\circ} \mathrm{C}$ for $5 \mathrm{~min}$ followed by 35 cycles at $94{ }^{\circ} \mathrm{C}$ for $30 \mathrm{~s}, 47{ }^{\circ} \mathrm{C}$ for $30 \mathrm{~s}$, and $72{ }^{\circ} \mathrm{C}$ for $1 \mathrm{~min}$ $30 \mathrm{~s}$, and a final step at $72{ }^{\circ} \mathrm{C}$ for $10 \mathrm{~min}$. The purified PCR product was sequenced in an ABI PRISM $^{\circledR} 377$ automated sequencer (Applied Biosystems, Foster City, California). The sequences are available from GenBank (accession Nos. EF126758-EF126759). 


\section{Larva and pupa of Galagete protozona}

\section{Description}

\section{Larva (Figs. 2-12)}

Length 5.0-11.6 $\mathrm{mm}(n=51),<5.0 \mathrm{~mm}(n=$ 16). Body pale gray, with microconvolutions; head capsule amber; prothoracic shield amber, gradually darkening posteriorly; pinacula pale brown; anal plate pale amber; setae with widened, circular, and slightly raised sockets. Head (Figs. 2-5, 12): hypognathous, granulose (Figs. 2-3); adfrontal sclerites widened distally, AF1 and AF2 short and equal in length, AF2 above apex of frons and AF1 below; F1, C1, and $\mathrm{C} 2$ about equal in length, at least 4 times longer than AF setae; F1 slightly closer to AF1 than to $\mathrm{C} 1$; clypeus with 6 pairs of setae, 3 pairs on medial half, 3 on lateral half; mandible (Fig. 12) with rounded outer edge, with small apical dentition, and with one large, narrow dentition on inner surface, bearing pair of subequal setae on outer surface near condyle; sensilla types and arrangement on antenna (Fig. 4) and on maxillary palp (Fig. 5) similar to those of other gelechioids (for references see Landry et al. 2006). Three stemmata in genal area, a proximate pair dorsolateral to antenna, and one stemma below antenna (stemmata 1, 2, and 6 absent); substemmatal setae about equal in length, arranged as in Fig. 3; S1 and S2 short, about equal in length, S3 about 2.5 times longer than $\mathrm{S}$ setae; $\mathrm{S} 3$ ventral to $\mathrm{S} 2$, S2 proximate to stemma 3 , and $\mathrm{S} 1$ proximate to stemma 5; A-group setae above gena, mesal to L1; P1 dorsolateral to AF2, P2 dorsomesal to P1. Thorax (Figs. 6, 9): T1 with $\mathrm{L}$ group trisetose, on large pinaculum extended beneath and anterior of spiracle; setae anterior to spiracle; L1 proximate and posteroventral to L2, about 2 times length of L2 and 4 times length of L3; L2 about equal distance from L1 and L3; SV-group setae on anterior part of elongate pinaculum; SV1 about 1/3 longer than SV2; SV1 in straight line with SV2; coxae nearly touching, V1s very proximate (not shown); segments of leg smooth, with minute spines dorsally on tibia, claw single (Fig. 6); shield with SD1 slightly anterior to and slightly longer than XD2 and $\mathrm{XD} 1$; XD2 and XD1 equal in length and about $1 / 3$ longer than D1; D1 and SD2 about equal in length; XD2 about equal distance from XD1 and SD1; D1 in straight line with XD1, slightly posterior to SD2 and D2; D2 about as long as SD1, in straight line with SD2. T2-T3 (Fig. 9): D2 about 4 times length of D1, both on small pinaculum; SD1 almost 4 times length of SD2, both on small pinaculum; L1 about 2 times length of L2, both on small pinaculum; L3 slightly shorter than L2, posterior to or in vertical line with SV1; MV1 on anterior margin of T2 and T3, slightly above SV1 (hard to see); $\mathrm{V} 1 \mathrm{~s}$ on T2-T3 about equal distance apart, at least 4 times distance between V1s on T1 (not shown). Abdomen (Figs. 7, 8, 10, 11): A1-A2 (Fig. 10): D2 about 2.5-3 times longer than D1, MD1 on anterior part of segment anteroventral to $\mathrm{D} 1$; SD1 above spiracle, about $1 / 3$ longer than D2, with minute SD2 (anterior part of pinaculum); a small opening on ventroposterior margin of pinaculum bearing SD1 and SD2; spiracle on A1 slightly larger than those on A2A7; L1 2 times length of L2, both on same pinaculum, aligned with or slightly posterior of spiracle; L3 about 3 times length of L2, anterior to, in vertical line with, or posterior to D2; SV group bisetose and separate on A1, trisetose on $\mathrm{A} 2$, and on same pinaculum; V1s equal distance apart (not shown). A3-A10 (Figs. 7, 8, 11): A3-A6 with 4 pairs of protuberant prolegs, crochets biordinal, in circle (Fig. 7); setae as on A2; A7 like A2 except SV group bisetose and on same pinaculum; A8 like A2 except with spiracle slightly larger and SV group unisetose; A9 with D2 about 2.5-3 times longer than D1; D1 anterior to D2, in straight line with it, in vertical line with and closer to SD1, SD1 more than 2 times length of D1; L1 and L2 on same pinaculum, slightly anterior to D1; L1 almost 5 times length of L2; L3 about as long as L2; L1 about 2 times length of SV1; V1s as on previous segments. A10 (Figs. 8, 11): anal plate with SD2, SD1, and D2 roughly equal in length, about 3 times length of D1; crochets of proleg biordinal, in semicircle, gradually shortened mesally and laterally.

\section{Pupa (Figs. 13-16)}

Length 5.0-6.7 mm $(n=4)$. Amber, smooth, all setae apically hooked. Sclerites of antennae annulated, widely separated anteriorly, gradually convergent from beyond basal $1 / 3$ of sclerites of maxillae, fused near apices of sclerites of maxillae, gradually divergent posteriorly, exposing distal part of sclerites of hindlegs; sclerites of midlegs not fused distally; paired nodular scars of prolegs on A5-A6 (Fig. 13); 
Figs. 2-8. Scanning electron micrographs of the larva of Galagete protozona. 2, Frontolateral view of head capsule. 3, Ventrolateral view of head capsule. 4, Sensilla of antenna: 1, sensilla basiconica; 2, sensillum chaetica; 3, sensillum styloconicum; 4, sensillum trichodeum. 5, Sensilla of maxillary palpus: A2, sensillum styloconicum; A1, A3, M1-2, L1-3, sensilla basiconica; SD, sensillum digitiform. 6, Distal portion of left prothoracic leg showing claw. 7, Left proleg on A5. 8, Anal plate of A10. Scale bars $=100 \mu \mathrm{m}$ (Figs. 2, 3, 68) and $10 \mu \mathrm{m}$ (Figs. 4, 5).

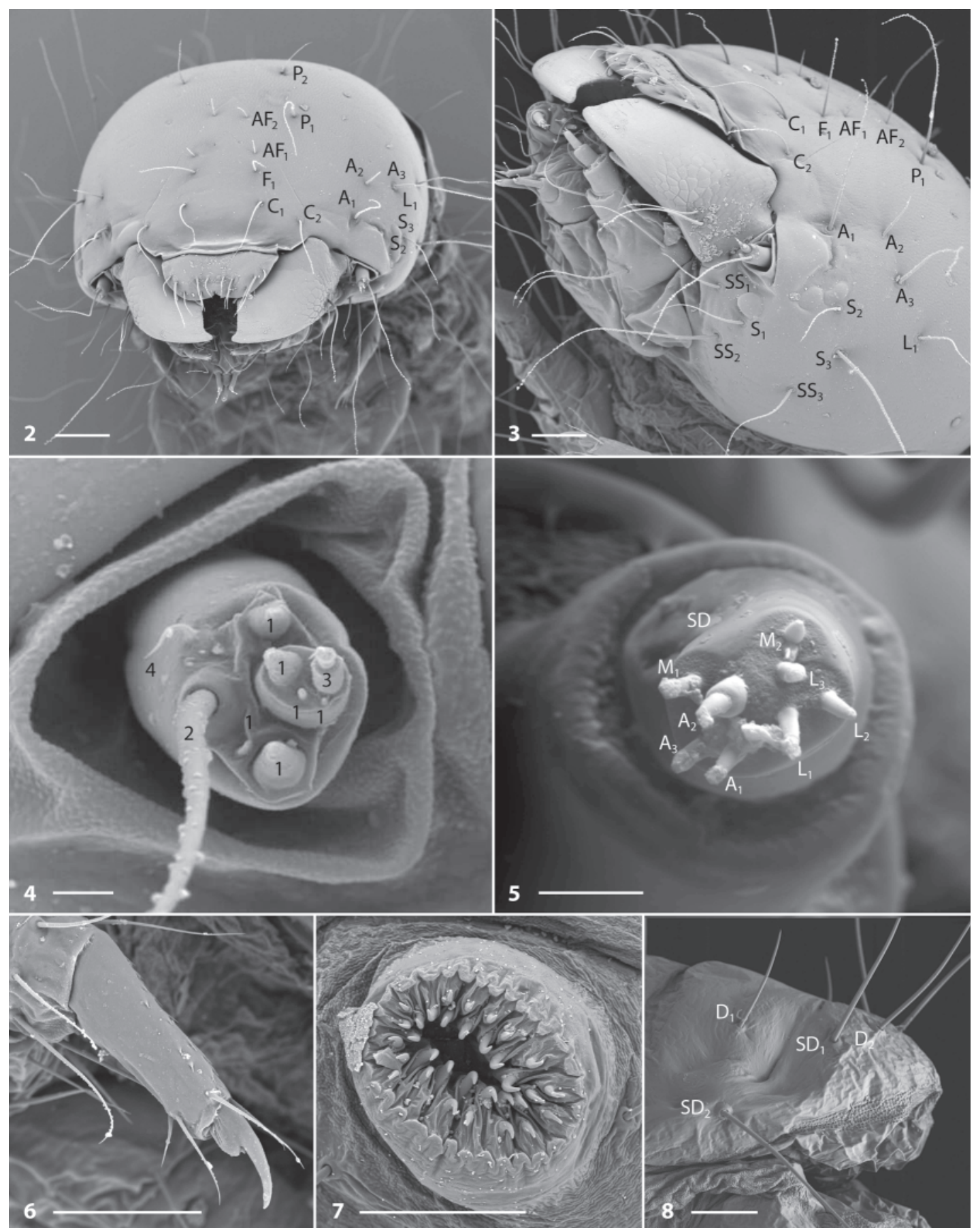

(c) 2007 Entomological Society of Canada 
Figs. 9-12. Chaetotaxy of the larva of Galagete protozona: 9, thorax; 10, abdominal segments 1-2; 11, abdominal segments 6-10;12, mandible.

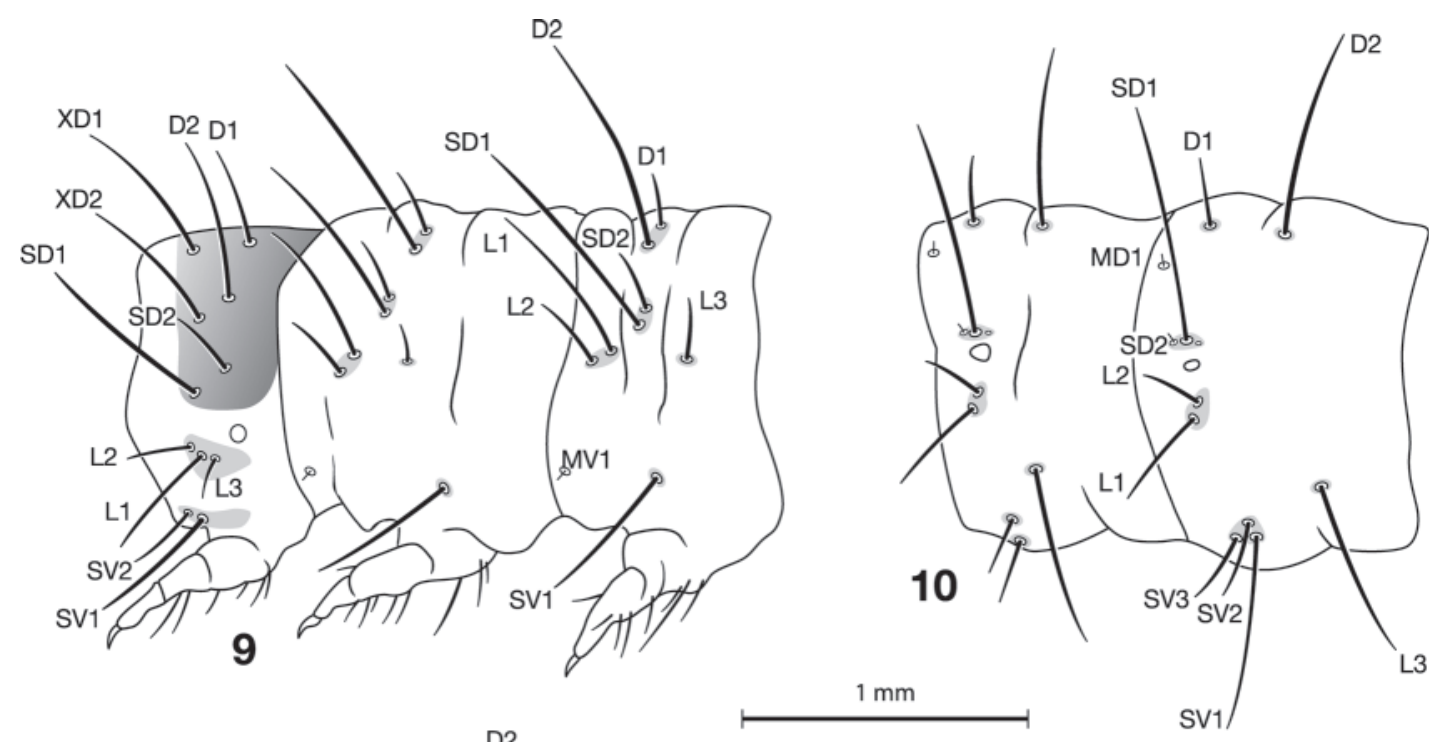

11
SV1

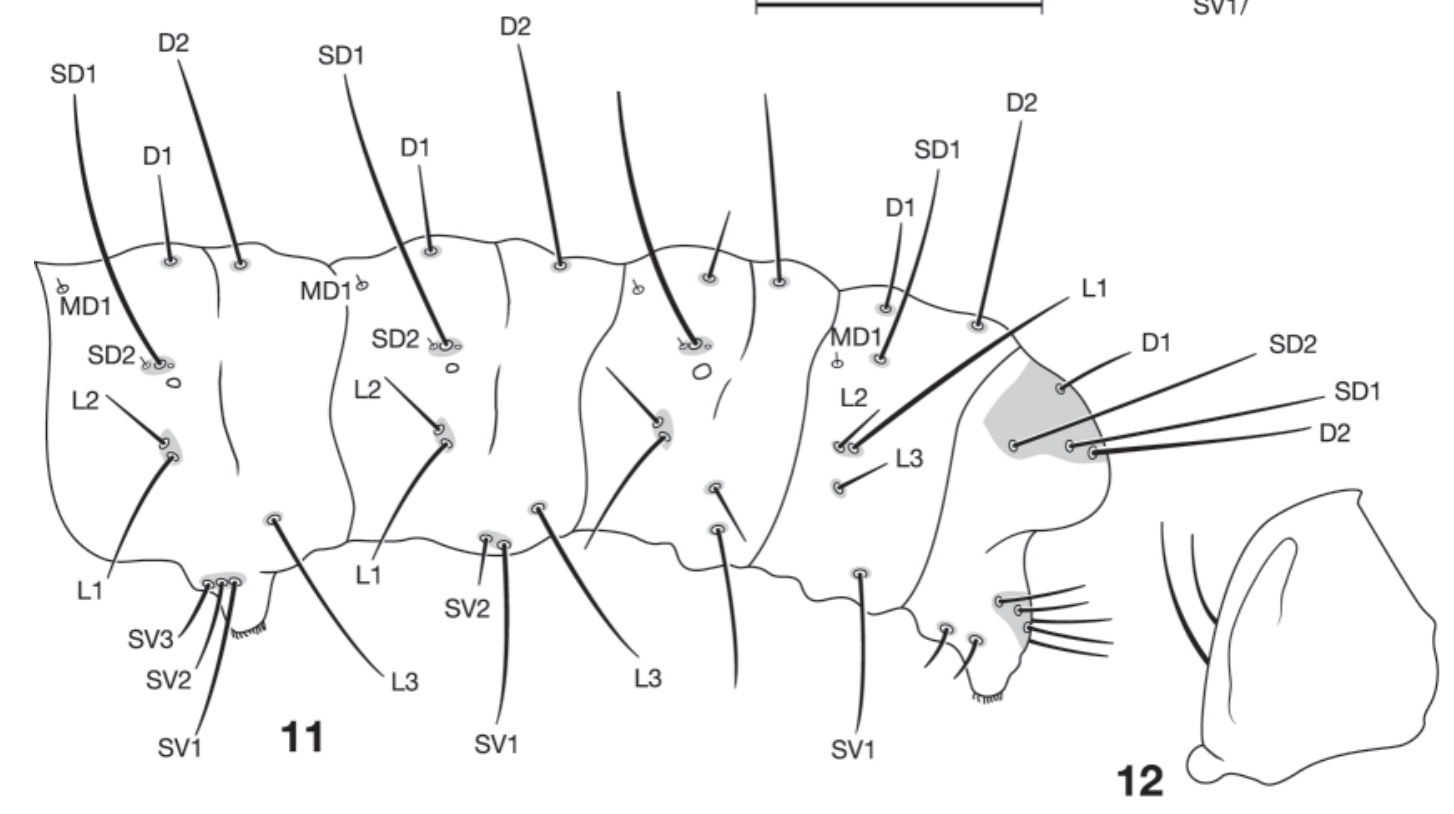

$\stackrel{0.1 \mathrm{~mm}}{\longmapsto}$
A6-A10 fused, rotating as a unit; cremaster dorsolaterally flattened, extended posterolaterally into two slightly divergent and elongate spine-like processes (Figs. 15, 16).

\section{Biology}

The small pinkish eggs are laid underneath dry iguana droppings. The Galapagos land iguana is mainly herbivorous but feeds on animal matter if easily accessible (Werner 1982).
In the dry fecal matter we found numerous seeds, leaves, and other plant material, but also remains of the large endemic painted locust, Schistocerca melanocera (Stål, 1860) (Acrididae), and once also iguana skin (probably from moulting). Therefore, the larvae may feed on dead plant and animal material, which is consistent with the feeding habits of other Autostichidae (Hodges 1998). In February 2005, adults were attracted to light on 


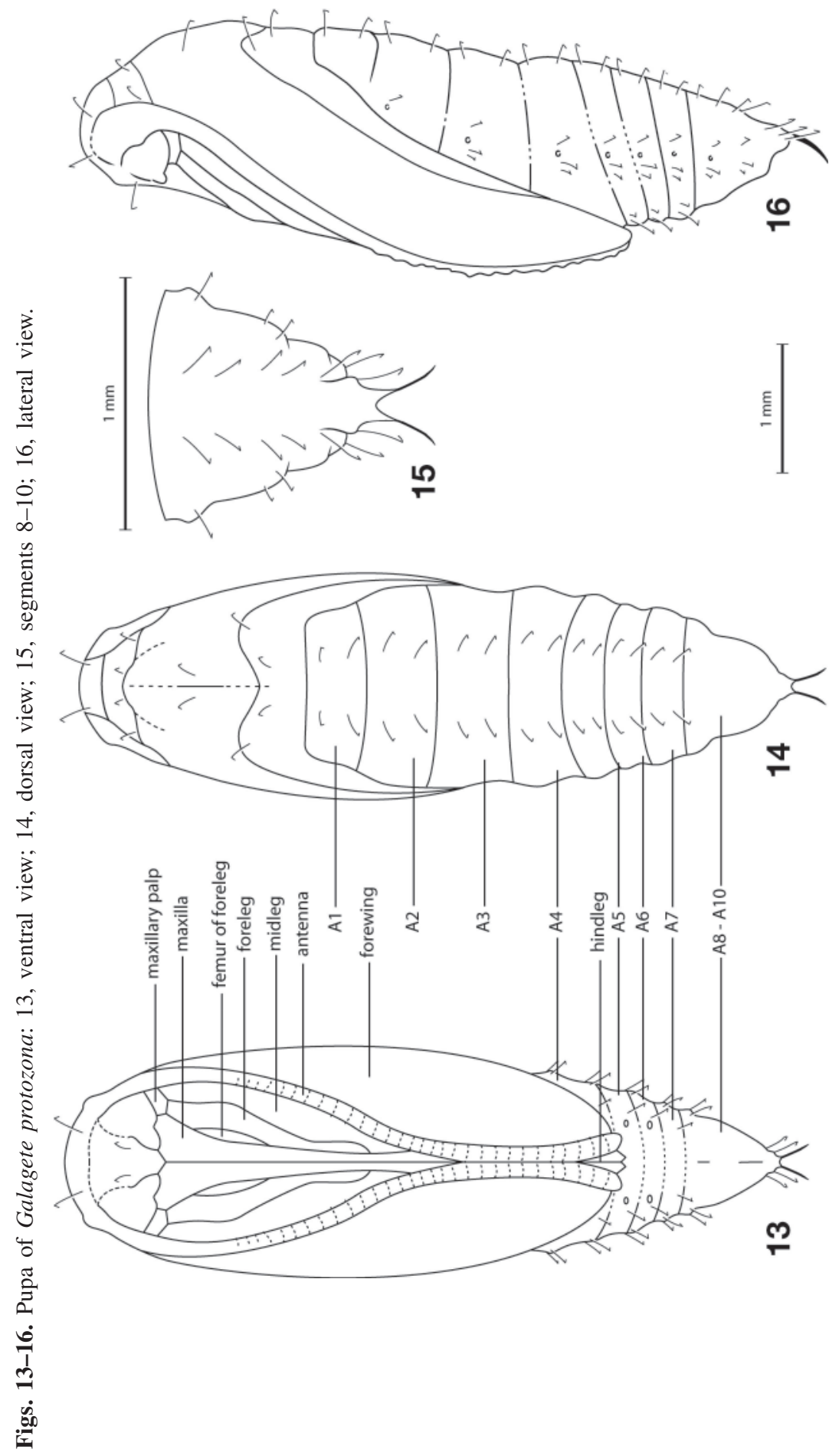

(C) 2007 Entomological Society of Canada 
Fernandina from sea level to the crater rim, which culminates at an elevation of $1341 \mathrm{~m}$.

\section{Relationships}

The main differences between larvae of $G$. protozona and those of the closely related species Taygete sphecophila (Meyrick, 1936), also known from the Galapagos (Landry et al. 2006), are as follows: mandible less angular, without subapical notch, and with smaller and narrower dentition on inner surface; position of S3 on head capsule more mesal; microsculpture of larval head different (that of $T$. sphecophila has slightly raised polygonal ridges); thoracic legs less spiny; D2 longer than D1 on T2-T3; L3 much longer than L2 on A1-A7 (3 times longer versus $1 / 3$ longer in T. sphecophila); A9 with SD1 more than 2 times length of D1 (versus about as long); SD1 closer to D1 and in vertical line with it (versus equidistant between D1 and D2 and in vertical line with D2); L1 almost 5 times length of L2 (versus 3 times). In both species, the larvae possess only three stemmata, a condition that is highly unusual in Gelechioidea (Landry et al. 2006).

The pupae of $G$. protozona and $T$. sphecophila are also similar except for the presence of hooked setae ventrally as well as dorsally (T. sphecophila has them only dorsally), the antenna and hindleg sclerites reaching beyond the apical margin of A4 (they just reach A4 on T. sphecophila), and the longer and curved (versus straight) processes at the apex of the abdomen.

\section{Notes}

Known only from the Galapagos, G. protozona is present on the islands of Baltra, Fernandina, Floreana, Isabela, Santa Cruz, Santa Fe, and Seymour Norte. The actual distribution of the Galapagos land iguana (Conolophus subcristatus) is on the islands of Baltra, Fernandina, Isabela, Plaza Sur, Santa Cruz, and Seymour Norte. It became extinct on Santiago and very restricted to isolated localities on Santa Cruz. Another endemic species of land iguana, Conolophus pallidus Heller, 1903, occurs only on the island of Santa Fe. Although the presence of $G$. protozona matches closely the distribution of these iguanids in the Galapagos, an existing specificity is not confirmed yet.

The COI gene sequences of a larva of G. protozona from Baltra and an adult from Seymour Norte showed no substitutions, which clearly indicates conspecificity. These sequences have been added to a data set that includes sequences of other Galagete species and that is being used to reconstruct the phylogeny of the species and populations of Galagete to get insight into the early stages of their speciation (unpublished data).

\section{Acknowledgements}

We are grateful to the authorities of the Galapagos National Park for permits, to Lazaro Roque-Albelo and other staff of the Charles Darwin Research Station, Galapagos, for logistical support, and to L. Roque, Jill Key, and Novarino Castillo for companionship and help in the field. We thank André Piuz, manager of the Scanning Electron Microscopy Laboratory of the MHNG, for preparing the larval specimens for study and for the electron micrographs (Figs. 2-8) and Florence Marteau, scientific illustrator at MHNG, for the digital illustrations and production of the plates of the larva and pupa (Figs. 9-16). Finally, we thank the Augustin-Lombard Foundation, the E. and L. Schmidheiny Foundation, the Swiss Academy of Sciences, the Baslerstiftung für biologische Forschung, the Department of cultural affairs of the city of Geneva, and the MHNG for travel funds, and two anonymous reviewers for their comments on the manuscript.

\section{References}

Hodges, R.W. 1998. The Gelechioidea. In Handbook of zoology, Lepidoptera, moths and butterflies, Vol. 1: Evolution, systematics, and biogeography. Edited by N.P. Kristensen. Walter de Gruyter, Berlin and New York. pp. 131-158.

Landry, B. 2002. Galagete, a new genus of Autostichidae representing the first case of an extensive radiation of endemic Lepidoptera in the Galapagos Islands. Revue suisse de zoologie, 109: 813-868.

Landry, B., Adamski, D., Schmitz, P., Parent, C., and Roque-Albelo, L. 2006. Taygete sphecophila (Meyrick) (Lepidoptera; Autostichidae): redescription of the adult, description of the larva and pupa, and impact on Polistes wasps (Hymenoptera; Vespidae) nests in the Galapagos Islands. Revue suisse de zoologie, 113: 307-323.

Meyrick, E. 1926. On Micro-Lepidoptera from the Galapagos Islands and Rapa. Transactions of the Royal Entomological Society of London, 74: 269278. 
Stehr, F. 1987. Superfamily Gelechioidea. In Immature insects, Vol. 1. Edited by F. Stehr. Kendall/Hunt Publishing Company, Dubuque, Iowa. pp. 379-399.

Werner, D.I. 1982. Social organization and ecology of land iguanas, Conolophus subcristatus, on Isla
Fernandina, Galapagos. In Iguanas of the world: their behavior, ecology, and conservation. Edited by G.M. Burghardt and A.S. Rand. Noyes Publications, Park Ridge, New Jersey. pp. 342-365. 